\title{
Critical Shoulder Angle and Its Clinical Correlation in Shoulder Pain
}

\author{
Kishore Vellingiri ${ }^{1}$, Prabhu Ethiraj ${ }^{1}$, Arun H. Shanthappa ${ }^{1}$ \\ 1. Department of Orthopaedics, Sri Devaraj Urs Medical College, Sri Devaraj Urs Academy of Higher Education \& \\ Research, Kolar, IND
}

Corresponding author: Prabhu Ethiraj, prabhu.thepreacher@gmail.com

\section{Abstract \\ Introduction}

The critical shoulder angle is defined as the angle formed between the plane of the glenoid and the line connecting the most lateral border of the acromion process, as seen on the true anteroposterior radiograph of the shoulder. The purpose of this study was to determine the association between the critical shoulder angle and shoulder pathologies like rotator cuff tears and glenohumeral osteoarthritis. It was also to assess the reproducibility and accuracy of critical shoulder angle values, which were measured with radiographs.

\section{Objective}

The aim of the study was to find out the association between the critical shoulder angle and shoulder injuries in a rural population cohort. The secondary aims were to assess clinical and radiological correlations between the critical shoulder angle and the symptomology of shoulder pain.

\section{Materials and methods}

Our study analysis was a prospective design conducted at R L Jalappa Hospital \& Research Centre, Karnataka, South India. After meeting the inclusion and exclusion criteria, 100 patients were recruited for the study. Forty-five patients had glenohumeral osteoarthritis and 55 patients had a diagnosis of rotator cuff tears. The majority of the patients were male $(70 \%)$ in both the glenohumeral osteoarthritis and rotator cuff tear groups. The mean critical shoulder angles in the glenohumeral osteoarthritis and rotator cuff tear groups were 30.31 and 33.62 , respectively.

\section{Conclusions}

Our data aid in demonstrating that glenohumeral osteoarthritis is associated with a significantly narrower critical shoulder angle and wider critical shoulder angles in rotator cuff disease. Further studies, however, should determine whether this association has a cause-and-effect relationship.

Received 07/29/2020

Review began 08/04/2020 Review ended 08/05/2020 Published 08/17/2020

\section{() Copyright 2020}

Vellingiri et al. This is an open access article distributed under the terms of the Creative Commons Attribution License CC-BY 4.0., which permits unrestricted use, distribution, and reproduction in any medium, provided the original author and source are credited.
Categories: Radiology, Orthopedics

Keywords: critical shoulder angle, glenohumeral osteoarthritis, rotator cuff tears

\section{Introduction}

The critical shoulder angle is the angle formed between the glenoid fossa plane (the line from the glenoid's inferior edge to the superior edge of the glenoid) and a line drawn from the inferior edge of the glenoid to the lateral edge of the acromion on a true anteroposterior (Grashey) shoulder radiograph [1]. Wider critical shoulder angle values are usually associated with a full thickness rotator cuff tear whereas small critical shoulder angle values are usually associated with glenohumeral osteoarthritis.

Various biomechanical research shows that shoulder abduction, glenoid compression, and joint shear forces are dependent on the critical shoulder angle, which explains various stress and wear patterns seen in the shoulder joint [2]. Measurement of the critical shoulder angle in proper anteroposterior radiography is needed so as to adjust for the errors that may arise from different positioning of the scapula and acromion.

This present study envisages investigating the incidence of a shoulder injury and the association of the critical shoulder angle in a rural population. We also hope to assess the clinical and radiological correlation of the critical shoulder angle in patients with shoulder pain.

\section{Materials And Methods}

This prospective study was conducted by the department of orthopedics at R L Jalappa Hospital \& Research Centre, an affiliate of Sri Devaraj Urs Medical College, Tamaka, Kolar, Karnataka, South India, from August 2019 to February 2020 This study was approved by the institutional ethics committee. One 


\section{Cureus}

hundred patients were included in this study. The inclusion criteria were as follows: age between 40 and 60 years, isolated non-traumatic full-thickness rotator cuff tears, and isolated non-traumatic osteoarthritis. The exclusion criteria included the following: traumatic rotator cuff tears, post-traumatic osteoarthritis, prior surgery, and history of shoulder dislocation or subluxation. Patient demographics, shoulder pain history, and critical shoulder angle assessment using radiological means were recorded. The collected data was encoded and entered into a database. All the quantitative measures were expressed using mean and standard deviation. The significance of the difference in means between the two groups was analyzed using the chi-square test. P-value $<0.05$ was considered statistically significant.

\section{Measurements}

The critical shoulder angle is the angle formed between the glenoid fossa plane (the line from the glenoid's inferior edge to the superior edge of the glenoid) and a line drawn from the inferior edge of the glenoid to the lateral edge of the acromion on a true anteroposterior (Grashey) shoulder radiograph (Figure 1). An increased critical shoulder angle $\left(35^{\circ}\right)$ is thought to alter deltoid vectors, which results in increased superior shear forces on the rotator cuff muscles [1]. This increased loading of the rotator cuff is a risk factor in developing rotator cuff tears. A reduced critical shoulder angle $\left(30^{\circ}\right)$ is associated with glenohumeral arthritis as the increased compressive forces acting across the glenohumeral joint [1].

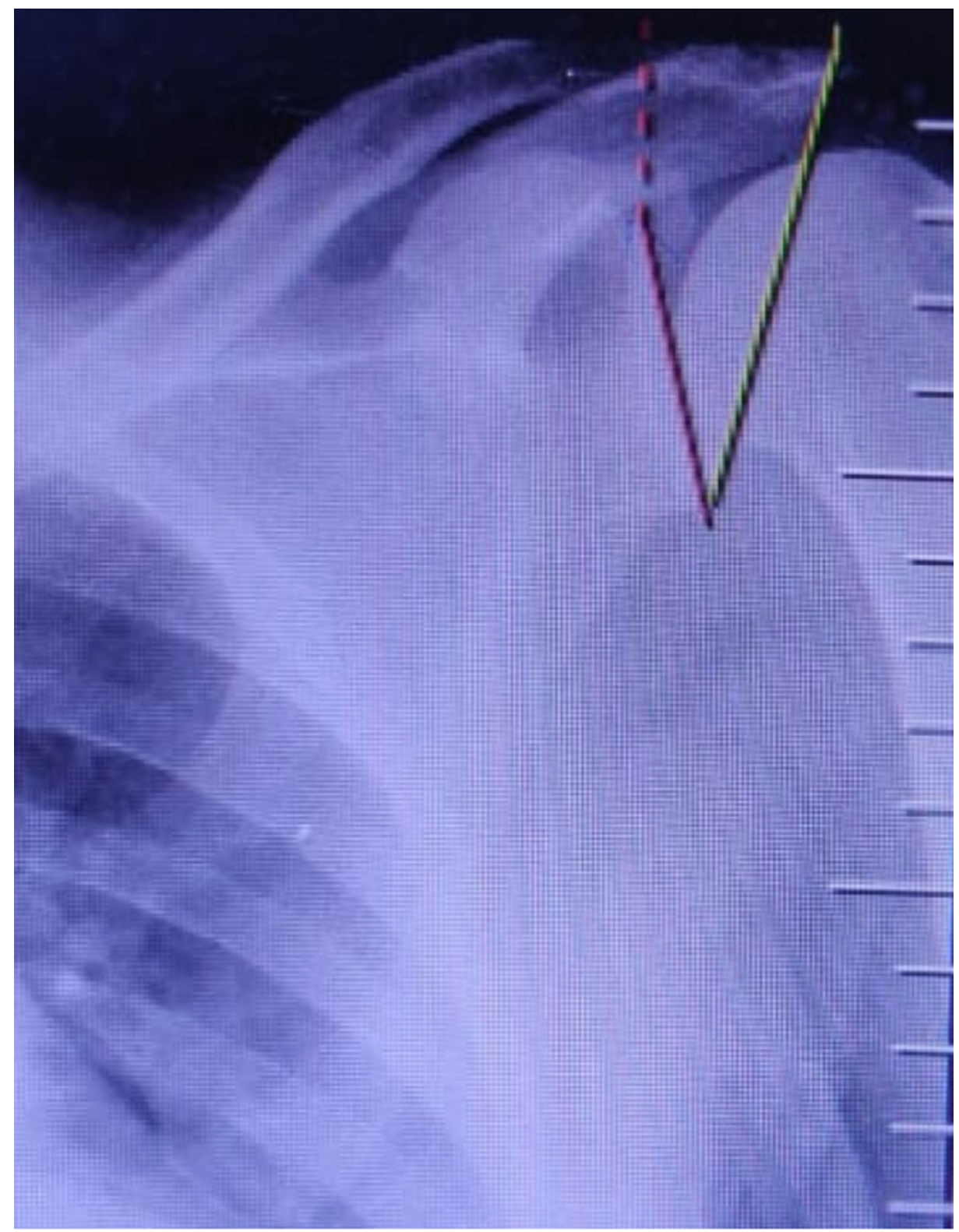

\section{FIGURE 1: Critical shoulder angle measurement on the AP view}




\section{Cureus}

\section{Results}

Our study was done on 100 patients of which $30 \%$ were female and the rest of the $70 \%$ were males. Thirtyfive (35\%) were left-sided and sixty-five (65\%) were right-sided. The age group distribution was $40-50$ years in 34 (34\%), 51-60 years in 31 (31\%), 61-70 years in 28 (28\%), and more than 70 years of age in seven (7\%) patients. Most of the patients were male (70\%) in both the osteoarthritis and rotator cuff tear groups. Fortyfive patients had glenohumeral osteoarthritis and 55 patients were made a diagnosis of rotator cuff tears (Figures 2-3). The mean critical shoulder angles in the osteoarthritis and rotator cuff tears groups were 30.31 and 33.62, respectively. Tables $1-3$ show the statistical significance of gender distribution, mean critical shoulder angle, and the correlation between age and the critical shoulder angle, respectively. Figures 4-5 show the scatter plot diagrams.

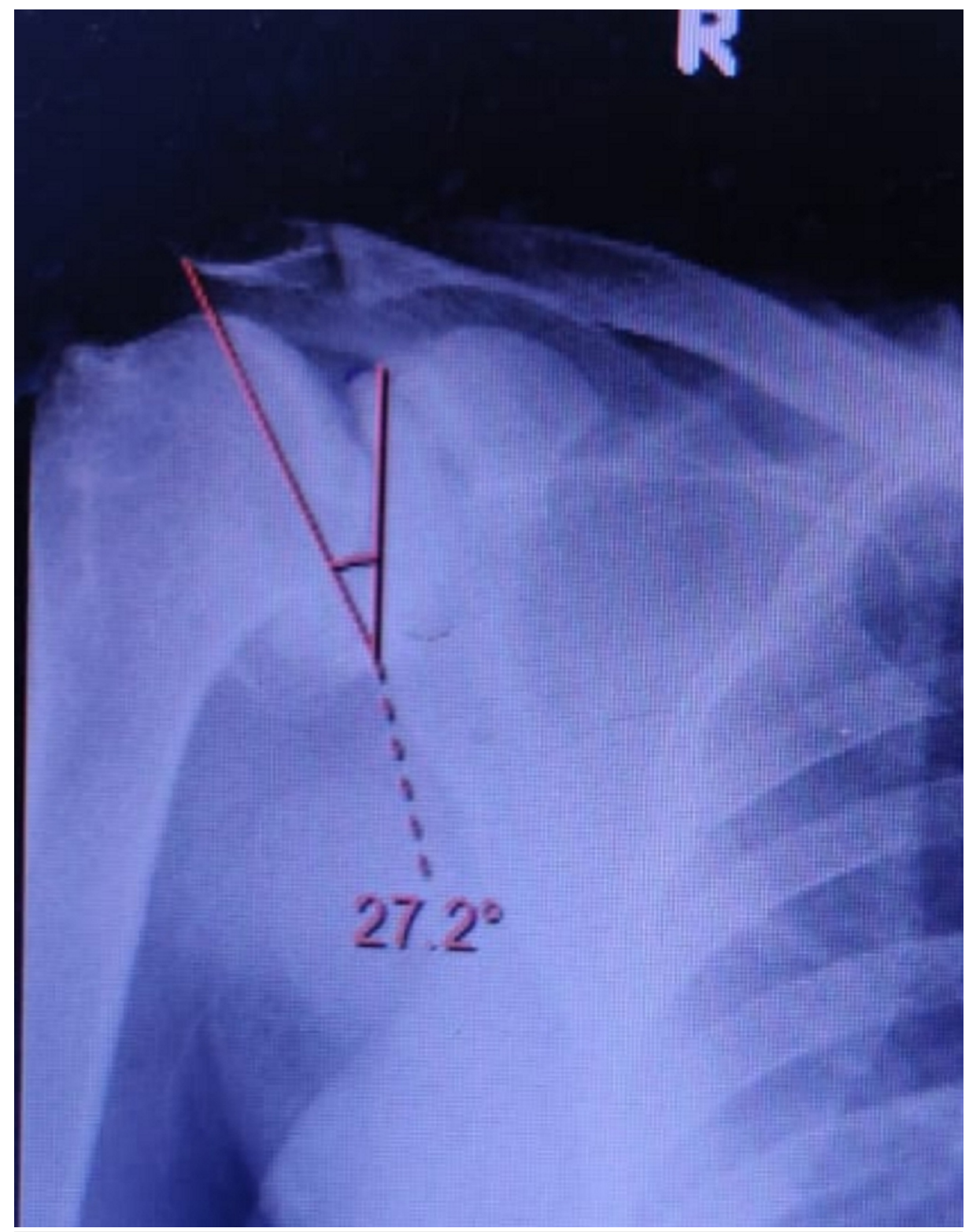

FIGURE 2: Critical shoulder angle measuring 27.2 degrees in a glenohumeral osteoarthritis patient 


\section{Cureus}

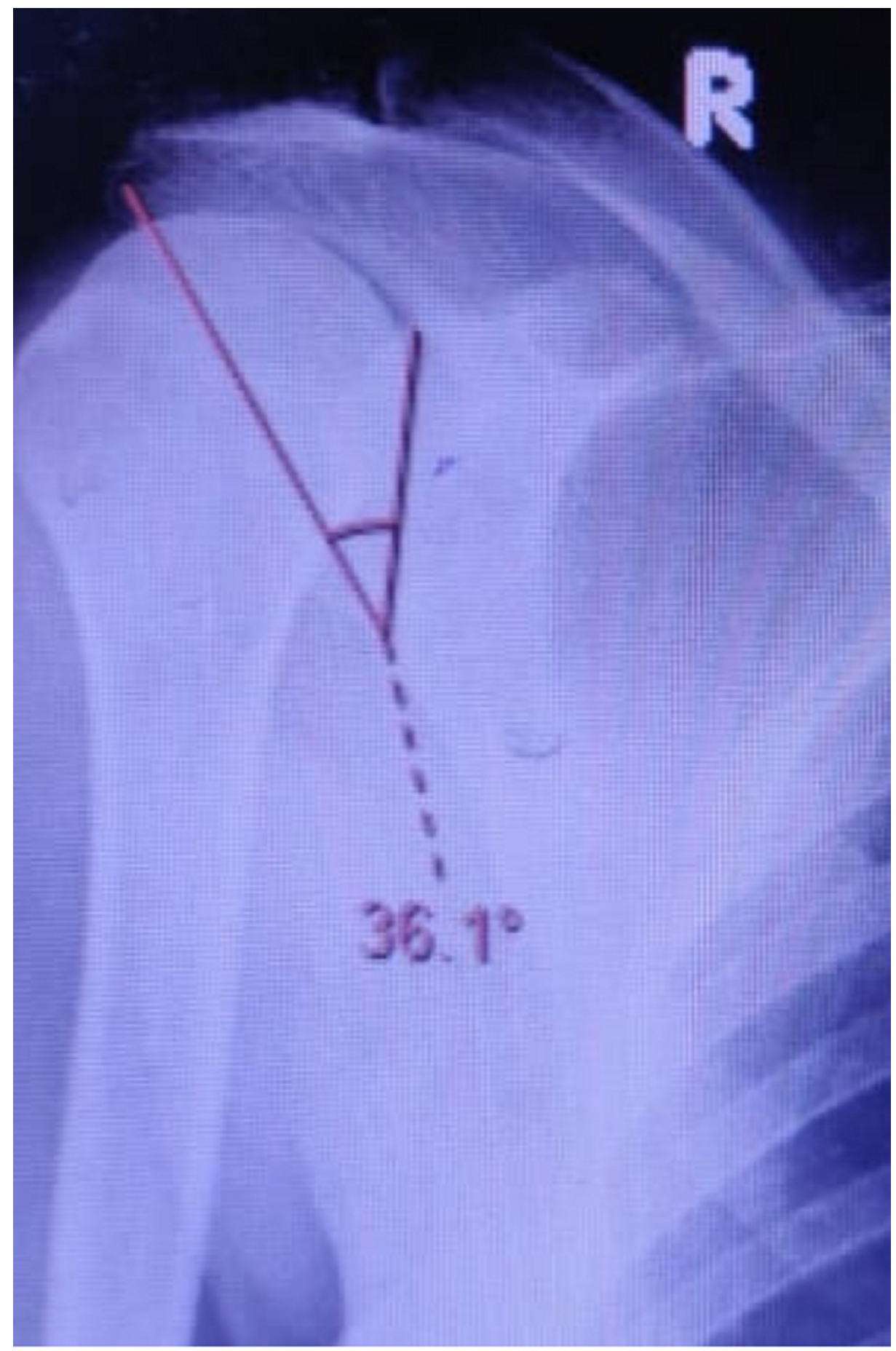

FIGURE 3: Critical shoulder angle measuring 36.1 degrees in a rotator cuff tear patient 


\section{Cureus}

\begin{tabular}{|c|c|c|c|c|c|}
\hline & & \multicolumn{2}{|c|}{ Groups } & \multirow{2}{*}{ Chi-square value } & \multirow{2}{*}{ P-value } \\
\hline & & OA & RCT & & \\
\hline \multirow{2}{*}{ Gender } & $\mathrm{F}$ & 15 & 15 & \multirow{3}{*}{0.433} & \multirow{3}{*}{0.511} \\
\hline & M & 30 & 40 & & \\
\hline Total & & 45 & 55 & & \\
\hline
\end{tabular}

TABLE 1: Chi-square value and $p$-value showing that gender distribution is not statistically significant between the osteoarthritis and rotator cuff tear groups

OA: osteoarthritis; RCT: rotator cuff tear

\begin{tabular}{|c|c|c|c|c|c|c|}
\hline & Groups & $\mathbf{N}$ & Mean & Std. Deviation & t-value & p-value \\
\hline \multirow{2}{*}{ CSA } & OA & 45 & 30.31 & 1.125 & \multirow{2}{*}{11.170} & \multirow{2}{*}{$<0.01$} \\
\hline & RCT & 55 & 33.62 & 1.705 & & \\
\hline
\end{tabular}

TABLE 2: Difference in the mean critical shoulder angle between the osteoarthritis and rotator cuff tear groups was found to be statistically significant ( $p$-value $<0.01$ )

OA: osteoarthritis; RCT: rotator cuff tear; CSA: critical shoulder angle

\begin{tabular}{|c|c|c|c|}
\hline Groups & Variables & r-value & p-value \\
\hline OA & & 0.22 & 0.146 \\
\hline RCT & & 0.82 & 0.55 \\
\hline
\end{tabular}

TABLE 3: Correlation between age and critical shoulder angle in both the groups

Though there is a positive correlation, it is not statistically significant. As age advances, the chance of acquiring the diseases will be high.

OA: osteoarthritis; RCT: rotator cuff tear; CSA: critical shoulder angle 


\section{Cureus}

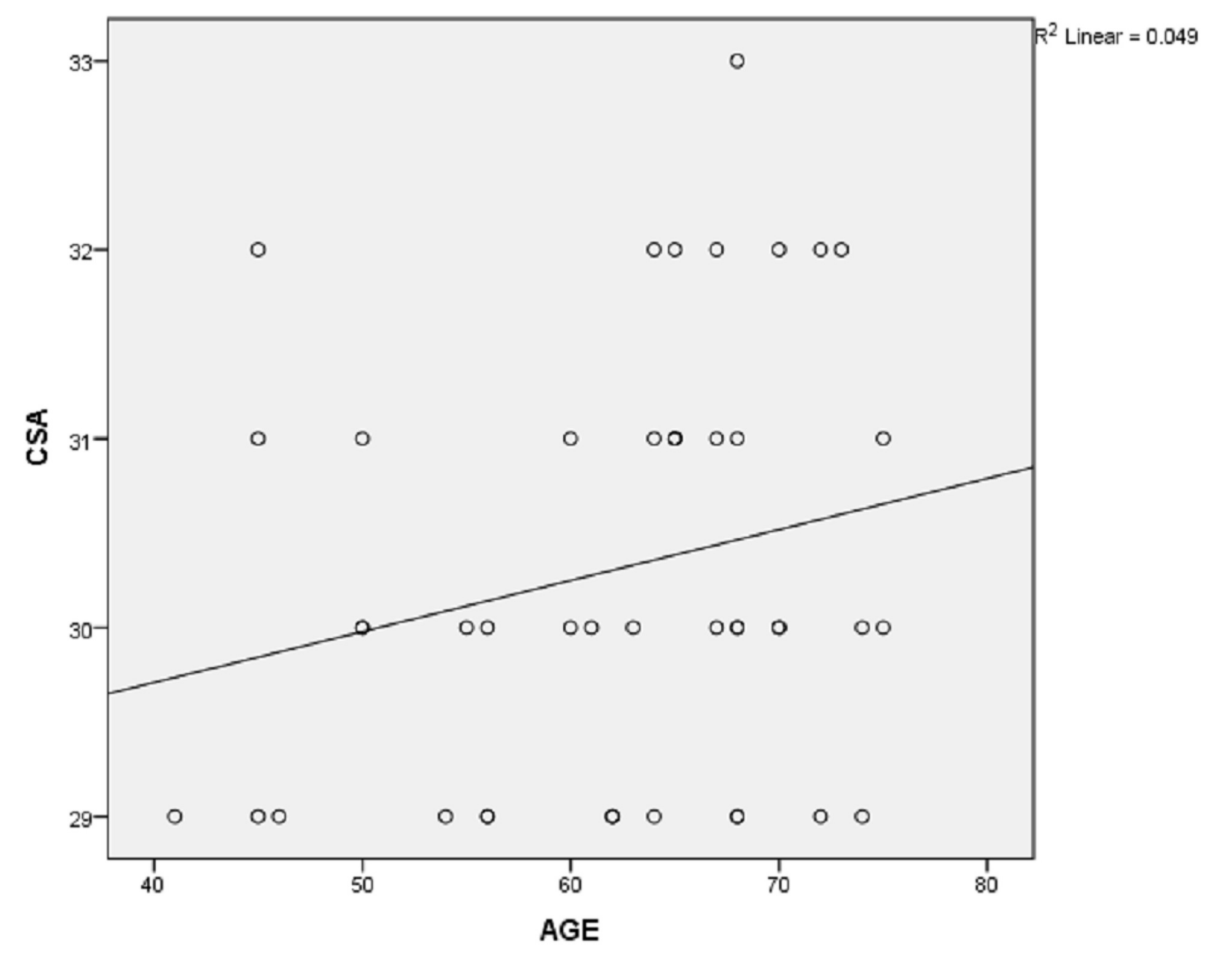

FIGURE 4: Scatter plot diagram showing a positive correlation between age and critical shoulder angle in the osteoarthritis group

CSA: critical shoulder angle

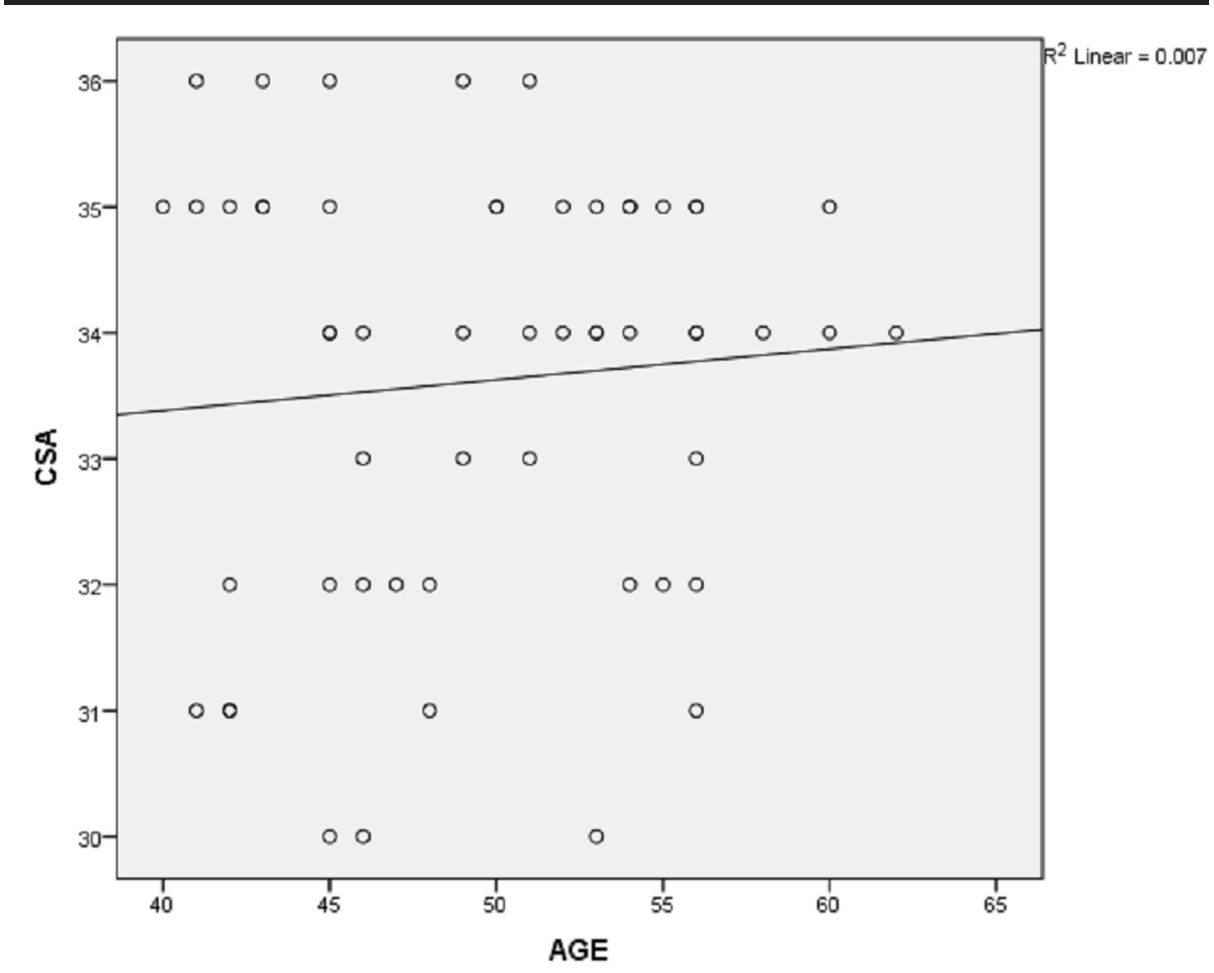

FIGURE 5: Scatter plot diagram showing a positive correlation between age and critical shoulder angle in the rotator cuff tear group 


\section{Discussion}

The critical shoulder angle would correlate with the wear of both the rotator cuff tendons or the articular cartilage of the glenohumeral joint. The critical shoulder angle is useful in helping surgeons determine when it is necessary to order a preoperative magnetic resonance imaging study in the evaluation of the rotator cuff. In the original paper, Moor B K et al. suggested that the mean critical shoulder angle was $33^{\circ}$ in a healthy shoulder control group, $28^{\circ}$ in patients having osteoarthritis, and $38^{\circ}$ in the rotator cuff tear group [1]. A larger acromial index was associated with an increased number of tendons torn and anchors used for repair, and it recorded more disability by the Quick Disabilities of the Arm, Shoulder and Hand Outcome Measure and poorer physical health as measured by the Short Form-12 Physical Component Summary score [3]. Critical shoulder measurements from the radiograph demonstrated an excellent interobserver agreement with less variability than critical shoulder angles from magnetic resonance imaging in osteoarthritis patients [4]. Larger critical shoulder angles are associated with increased risk of symptomatic cuff tears, larger cuff tears, and the severity of eccentric osteoarthritis [5]. De Jesus et al. in their study showed that the supplementary scapula position must not be more than $20^{\circ}$ of either external or internal rotation [6]. Deviations less than these threshold values were made sure as nonrelevant in previous studies. The advantage of the critical shoulder angle measurement is that it accounts for both glenoid inclination and the lateral extent of the acromion and has been validated in biomechanical models. The lateral acromial roof extension has a higher influence in the pathogenesis of a degenerative rotator cuff tear and concentric osteoarthritis than acromial height or glenoid inclination [7]. The glenoid orientation in the scapulae of shoulders with a full-thickness tear of the rotator cuff was the same as that in shoulders with an intact rotator cuff [8]. The glenoid inclination angle was greater in cadaver shoulders having full-thickness rotator cuff tears (98.6 degrees) than in shoulders without tears (91.0 degrees ) [9]. Rotator cuff re-tear rates were more in patients with more critical shoulder angles among comparative, non-randomized studies. Based on the previous studies, it is unclear as to whether lateral acromioplasty affects clinical outcomes as a function of a decreased postoperative critical shoulder angle. The structural or mechanical integrity of the lateral deltoid origin was not weakened by arthroscopic lateral acromioplasty and nor did a $5-\mathrm{mm}$ or a $10-\mathrm{mm}$ arthroscopic lateral acromioplasty significantly reduce the deltoid's failure load [10]. According to Nyffeler et al., the humeral head needed to be in a neutral position or up to a maximum of $20^{\circ}$ internal rotation for being included in the definition of a true anteroposterior radiograph [11].

Watson-Jones R [12] and Armstrong JR [13] have implicated the risk factor for developing rotator cuff disease is the acromion's lateral extension. Nakagawa $Y$ et al. noticed that in primary glenohumeral osteoarthritis, the incidence was around $0.4 \%$ in patients with orthopedic complications and $4.6 \%$ in patients who suffered from shoulder diseases [14]. The incidence was found to be more among women and older patients above 60 years of age. Minagawa $\mathrm{H}$ et al. demonstrated that the prevalence of asymptomatic rotator cuff tear in the general population was $22.1 \%$, of which one-half of all tears were in the 50 years of age, whereas it accounted for two-thirds of those over the age of 60 years [15]. An asymptomatic tear was twice as common as a symptomatic tear. Neer CS in a study identified that total shoulder joint surface replacement had good early outcomes as compared to that of patients treated with hemi-replacement arthroplasty with a Vitallium humeral-head prosthesis in a glenohumeral osteoarthritis condition [16]. Recovery of adequate strength was slow and continued fatigability was noted in the latter part. Hamada $\mathrm{K}$ et al. proposed the following causes that lead to cuff-tear arthropathy in massive cuff tear patients: (I) arm elevation in activities of daily living, (II) rupture of the long head of biceps tendon, (III) the abnormal fulcrum of the humeral head against the acromion and the coracoacromial ligament, and (IV) the weakness of external rotation [17]. Moor et al. introduced the critical shoulder angle, which is thought to be predictive of both rotator cuff disease and glenohumeral arthritis [1]. Thus, reviewing the historical acromial indices and measurements is instructive in understanding how the critical shoulder angle is useful as a tool for determining and predicting various abnormalities of the shoulder. Abnormal acromial morphology, especially lateral acromial extension, contributes to rotator cuff disease development by creating altered mechanical vectors that affect both compressive and shear forces. The critical shoulder angle has been linked in developing both rotator cuff tears and osteoarthritis. An increased critical shoulder angle $\left(35^{\circ}\right)$ is thought to result in increased superior shear forces on the rotator cuff muscles due to altered vectors of the deltoid, which may help to predict, and may be a risk factor for developing rotator cuff tears. A decreased critical shoulder angle $\left(30^{\circ}\right)$ is related with glenohumeral arthritis because of an increase in compressive forces across the joint. Published studies have evidence of both supporting and refuting these associations; the conflicting findings may be due to the lack of standardized radiographic methods for measuring the critical shoulder angle and/or measurement errors. Critical shoulder angle and age, two assessable variables, adequately help in the prediction of shoulder pathologies in patients with shoulder complaints. Using the prediction model in primary care may be considered, as severe shoulder pathologies might be detected at an earlier time, thus early adequate treatment by a specialist could be applied.

\section{Limitations of the study}

The primary limitation of our study was that it covers a small sample size for this prevalence-based study and was done at a single center. There was no control group in the present study. Prospective longitudinal cohort studies involving a standard and reproducible method of critical shoulder angle measurement are needed to evaluate the true relationship between the critical shoulder angle and shoulder disease. 


\section{Conclusions}

Our data aid in demonstrating that glenohumeral osteoarthritis is associated with a significantly narrower critical shoulder angle and wider critical shoulder angles in rotator cuff disease. Further studies, however, should determine whether this association has a cause-and-effect relationship. The measurement of the critical shoulder angle on a radiograph being less prone to interobserver variability than on magnetic resonance imaging and the correlation of wider angles with re-tears of the rotator cuff (hence its ability to predict re-tears) make this study significant. It is especially useful in a developing country like India, where patient affordability is an issue and expensive magnetic resonance imaging could be avoided if osteoarthritis/a rotator cuff tear seems more likely based on clinical and radiographic correlation.

\section{Additional Information \\ Disclosures}

Human subjects: Consent was obtained by all participants in this study. Institutional Ethics Committee, Sri Devaraj Urs Medical College, Kolar issued approval SDUMC/KLR/IEC/80/2019-20, Dated : 30/08/2019.

Animal subjects: All authors have confirmed that this study did not involve animal subjects or tissue. Conflicts of interest: In compliance with the ICMJE uniform disclosure form, all authors declare the following: Payment/services info: All authors have declared that no financial support was received from any organization for the submitted work. Financial relationships: All authors have declared that they have no financial relationships at present or within the previous three years with any organizations that might have an interest in the submitted work. Other relationships: All authors have declared that there are no other relationships or activities that could appear to have influenced the submitted work.

\section{References}

1. Moor BK, Bouaicha S, Rothenfluh DA, Sukthankar A, Gerber C: Is there an association between the individual anatomy of the scapula and the development of rotator cuff tears or osteoarthritis of the glenohumeral joint? A radiological study of the critical shoulder angle. Bone Joint J. 2013, 935:41. 10.1302/0301-620X.95B7.31028

2. Heuberer PR, Plachel F, Willinger L, et al.: Critical shoulder angle combined with age predict five shoulder pathologies: a retrospective analysis of 1000 cases. BMC Musculoskelet Disord. 2017, 18:259.

3. Ames JB, Horan MP, Van der Meijden OA, Leake MJ, Millett PJ: Association between acromial index and outcomes following arthroscopic repair of full-thickness rotator cuff tears. J Bone Joint Surg Am. 2012, 94:1862-1869. 10.2106/JBJS.K.01500

4. Bhatia S, Hsu A, Lin EC, Chalmers P, Ellman M, Cole BJ, Verma NN: Surgical treatment options for the young and active middle-aged patient with glenohumeral arthritis. Adv Orthop. 2012, 2012:846843. $10.1155 / 2012 / 846843$

5. Blonna D, Giani A, Bellato E, Mattei L, Caló M, Rossi R, Castoldi F: Predominance of the critical shoulder angle in the pathogenesis of degenerative diseases of the shoulder. J Shoulder Elbow Surg. 2016, 25:13281336. 10.1016/j.jse.2015.11.059

6. De Jesus JO, Parker L, Frangos AJ, Nazarian LN: Accuracy of MRI, MR arthrography, and ultrasound in the diagnosis of rotator cuff tears: a meta-analysis. AJR Am J Roentgenol. 2009, 192:1701-1707. 10.2214/AJR.08.1241

7. Cherchi L, Ciornohac JF, Godet J, Clavert P, Kempf JF: Critical shoulder angle: measurement reproducibility and correlation with rotator cuff tendon tears. Orthop Traumatol Surg Res. 2015, 102:559-562. 10.1016/j.otsr.2016.03.017

8. Kandemir U, Allaire R B, Jolly JT, Debski RE, McMahon PJ: The relationship between the orientation of the glenoid and tears of the rotator cuff. J Bone Joint Surg Br. 2006, 8888:1105-1109. 10.1302/0301620X.88B8.17732

9. Hughes RE, Bryant CR, Hall JM, et al.: Glenoid inclination is associated with full thickness rotator cuff tears . Clin Orthop Relat Res. 2003, 407: 86-91. 10.1097/00003086-200302000-00016

10. Marchetti DC, Katthagen JC, Mikula JD, et al.: The effects of arthroscopic lateral acromioplasty on the critical shoulder angle and the anterolateral deltoid origin: an anatomic cadaveric study. Arthroscopy. 2016, 32:569-575. 10.1016/j.arthro.2016.08.015

11. Nyffeler RW, Werner CM, Sukthankar A, Schmid MR, Gerber C: Association of a large lateral extension of the acromion with rotator cuff tears. J Bone Joint Surg Am. 2006, 88:800-805.

12. Watson-Jones R: Fractures and joint injuries. Fractures and Joint Injuries. Williams \& Wilkins, Baltimore; 1940. 2:

13. Armstrong JR: Excision of the acromion in treatment of the supraspinatus syndrome; report of 95 excisions . J Bone Joint Surg Br. 1949, 31:436-442.

14. Nakagawa Y, Hyakuna K, Otani S, Hashitani M, Nakamura T: Epidemiologic study of glenohumeral osteoarthritis with plain radiography. J Shoulder Elbow Surg. 1999, 8:580-584. 10.1016/s10582746(99)90093-9

15. Minagawa H, Yamamoto N, Abe H: Prevalence of symptomatic and asymptomatic rotator cuff tears in the general population: from mass-screening in one village. J Orthop. 2013, 10:8-12. 10.1016/j.jor.2013.01.008

16. Neer CS 2nd: Replacement arthroplasty for glenohumeral osteoarthritis. J Bone Joint Surg Am. 1974, 56:113.

17. Hamada K, Fukuda H, Mikasa M, Kobayashi Y: Roentgenographic findings in massive rotator cuff tears. A long-term observation. Clin Orthop Relat Res. 1990, 254:92-96. 